\title{
Escala diagramática para avaliação da severidade da mancha alvo da soja
}

\author{
Rafael M. Soares, Cláudia V. Godoy \& Maria Cristina N. de Oliveira \\ Embrapa Soja, 86001-970, Londrina, PR, Brasil \\ Autor para correspondência: Rafael M. Soares, e-mail: rafael@cnpso.embrapa.br
}

\begin{abstract}
RESUMO
A mancha alvo da soja, causada pelo fungo Corynespora cassiicola, é encontrada em praticamente todas as regiões de cultivo de soja do Brasil e pode causar perdas econômicas. Esse trabalho teve como objetivo elaborar e validar uma escala diagramática para avaliação da severidade da mancha alvo da soja. Para tal, foram coletados folíolos de soja com sintomas da doença, onde se mediu a severidade, determinando-se os limites mínimos e máximos, e os níveis intermediários da escala de acordo com a lei do estímulo visual de Weber-Fechner. A escala elaborada apresentou sete níveis de severidade: 1, 2, 5, 9, 19, 33 e 52\%. A validação foi realizada por oito avaliadores, sem experiência em quantificar essa doença, que estimaram a severidade de 40 folíolos com diferentes níveis de severidade da mancha alvo, sem e com o auxílio da escala. A utilização da escala diagramática resultou em melhor acurácia e precisão das estimativas realizadas, sendo uma ferramenta de fácil e rápida utilização, que pode ser adotada para auxiliar na avaliação da severidade da mancha alvo da soja.
\end{abstract}

Palavras-chave: Glycine max, Corynespora cassiicola, quantificação de doenças.

\section{ABSTRACT}

Diagrammatic scale for severity evaluation of soybean target spot

Soybean target spot, caused by Corynespora cassiicola, is found in practically all the soybean growing regions in Brazil and it can cause economic losses. The objective of this work was to elaborate and validate a diagrammatic scale to evaluate soybean target spot severity. Soybean leaflets with disease symptoms were collected and severity was measured, determining the minimum and maximum limits and the intermediate levels of the scale, according to Weber-Fechner's stimulus-response law. The elaborated scale had seven levels: $1,2,5,9,19,33$ and $52 \%$. The validation was performed by eight persons without experience with this disease quantification, who estimated the severity of 40 leaflets with different levels of target spot severity, with and without the use of the scale. The use of a diagrammatic scale resulted in higher accuracy and precision of the estimations performed, meaning that this can be considered a quick and easy tool to help evaluate soybean target spot severity.

Keywords: Glycine max, Corynespora cassiicola, disease assessment.

A mancha alvo da soja Glycine $\max$ (L.) Merr. é causada pelo fungo Corynespora cassiicola (Berk. \& M.A. Curtis) C.T. Wei. Este patógeno foi identificado pela primeira vez nos EUA em 1945 com o nome de Helminthosporium vignae. No Brasil, os primeiros registros foram no Mato Grosso em 1974 e no Paraná em 1976 (Almeida et al., 1976). Surtos severos, mas esporádicos, têm sido observados nas regiões mais frias do Sul e nas regiões altas dos Cerrados (Tecnologias ..., 2008). O fungo é encontrado em praticamente todas as regiões de cultivo de soja do Brasil, acreditando-se ser nativo e infectar um grande número de espécies de plantas. Pode sobreviver em restos de cultura e sementes infectadas, sendo essa uma forma de disseminação. Condições de alta umidade relativa e temperaturas amenas são favoráveis à infecção na folha. Os sintomas mais comuns são manchas nas folhas, com halo amarelado e pontuação escura no centro, que causam severa desfolha. Ocorrem também manchas na haste e na vagem.
O fungo pode infectar raízes, causando podridão radicular e intensa esporulação (Henning et al., 2005).

Várias estratégias são recomendadas para o controle da doença tais como: o uso de cultivares resistentes, o tratamento de sementes, a rotação/sucessão de culturas com milho e espécies de gramíneas e pulverizações com fungicidas (Almeida et al., 1997; Henning et al., 2005). A despeito destas recomendações de controle e da importância cada vez maior dessa doença, existem poucas informações sobre a eficiência de fungicidas para seu controle, bem como não se tem conhecimento de programas de melhoramento de soja que testem rotineiramente seus materiais quanto à resistência a mancha alvo. Neste contexto, a importância de realizar esses testes vem acompanhada da necessidade de ter ferramentas adequadas para avaliação/quantificação da doença. Segundo Nutter Jr \& Schultz (1995), essas ferramentas devem ser de fácil e rápida utilização em diversas situações, mas também proporcionar resultados 
acurados, precisos e reprodutíveis. A acurácia significa ter uma estimativa da quantidade de doença próxima do valor real e a precisão refere-se à variação ou repetibilidade de uma estimativa. No caso de doenças foliares como a mancha alvo, a severidade (porcentagem da área de tecido ou volume coberto com sintomas) tem sido a variável mais adequada para quantificação. Mas a quantificação da severidade, quando estimada visualmente, está sujeita a subjetividade e erros. O uso de escalas diagramáticas, corretamente elaboradas, pode minimizar essa subjetividade, pois estas representam ilustrações da parte da planta a ser avaliada com sintomas em diferentes níveis de severidade (Bergamin Filho \& Amorim, 1996).

Para a elaboração de escalas diagramáticas é importante que os limites mínimos e máximos destas correspondam aos ocorridos no campo. Também, a medida da intensidade da doença nas figuras da escala deve ser precisa e os níveis intermediários devem respeitar as limitações de acuidade visual humana, segundo a definição da lei do estímulo visual de Weber-Fechner (Angelotti et al., 2008; Bergamin Filho \& Amorim, 1996). Esse trabalho teve como objetivo elaborar uma escala diagramática para avaliação da severidade da mancha alvo da soja, seguindo a metodologia recomendada para se obter uma escala de comprovada eficiência na redução da subjetividade das avaliações.

A metodologia utilizada consistiu em coletar folíolos de soja com sintomas da doença em diferentes níveis de severidade. As coletas foram feitas tanto a campo, em plantas infectadas naturalmente, como em casade-vegetação, em plantas inoculadas com isolado de $C$. cassiicola. Os folíolos coletados foram fotografados com câmera digital e analisados no software Quant (Vale et al., 2003), que calculou a severidade da doença na superfície de cada folíolo. Considerando um valor mínimo e máximo de severidade, determinaram-se os valores intermediários de severidade da escala, baseados na lei de Weber-Fechner de estímulo visual, que diz que a acuidade visual é proporcional ao logaritmo da intensidade do estímulo. Foram utilizadas imagens reais de folíolos na confecção da escala e, para se obter o valor exato de alguns dos níveis intermediários de severidade, foram feitas colagens virtuais de lesões nos folíolos, utilizando o software Microsoft ${ }^{\circledR}$ Paint 5.1. A validação da escala foi feita por meio de uma apresentação eletrônica com 40 fotografias de folíolos apresentando diferentes níveis de severidade da doença. A apresentação foi avaliada por oito pessoas consideradas sem experiência na quantificação desta doença. Primeiramente, a avaliação foi feita sem a utilização da escala e, em seguida, com a utilização da mesma.

Para se determinar a acurácia e a precisão das avaliações, foram comparados os resultados obtidos pelos oito avaliadores, com e sem a escala, com a severidade real. Para isso, foi aplicado o método da análise de regressão linear (Charnet et al., 1999), considerando a inclinação da reta de regressão igual a uma constante 1 . O modelo da regressão e as hipóteses apropriadas para avaliar a severidade da mancha alvo em folíolos de soja, considerando uma distribuição de probabilidade normal, são os que seguem:

$$
\text { Seja o modelo } \quad Y_{i}=a+b \mathrm{x}+\varepsilon_{\mathrm{i}}
$$

em que,

$Y_{i}$ é a variável dependente ou a severidade obtida pelos avaliadores; $a$ é o intercepto ou o coeficiente linear; $b$ é o coeficiente angular ou inclinação da reta de regressão; $x$ é a variável independente ou a severidade real da mancha alvo e, $\varepsilon_{i}$ são os erros com distribuição normal, $N \approx\left(0, \sigma^{2}\right)$.

Os coeficientes a e b são constantes desconhecidas e que doravante serão denominados parâmetros da equação de regressão linear e $x$ é uma constante conhecida.

As hipóteses para estes parâmetros são:

$$
\begin{array}{lll}
H_{0}: \mathrm{a}=0 & & H_{0}: \mathrm{b}=1 \\
\mathrm{H}_{1}: \mathrm{a} \neq 0 & & \mathrm{H}_{1}: \mathrm{b} \neq 1
\end{array}
$$

A acurácia das estimativas dos avaliadores foi determinada pela aplicação do teste $t(\mathrm{P} \leq 0,05)$ aos coeficientes da regressão linear $\left(H_{0}: a=0\right.$ e $\left.H_{0}: b=1\right)$. A precisão das estimativas foi avaliada através do coeficiente de determinação $\left(\mathrm{R}^{2}\right)$ e pela variância dos erros absolutos. Os erros absolutos foram calculados subtraindo a severidade real da severidade estimada (Nutter Jr \& Schultz, 1995). As análises estatísticas foram realizadas com o auxílio do programa SANEST (Zonta et al., 1982).

O valor mínimo de severidade da escala diagramática foi de $1 \%$ e o máximo foi de $52 \%$, ficando a escala com sete níveis de severidade: $1,2,5,9,19,33$ e $52 \%$ da área do folíolo com lesão (Figura 1). Os valores estimados e o teste t para os parâmetros intercepto $(a)$ e coeficiente angular da reta $(b)$ e do coeficiente de determinação $\left(\mathrm{R}^{2}\right)$, com e sem utilização da escala diagramática, estão apresentados na Tabela 1. Os resultados do teste $t$ para os coeficientes lineares $(a)$, sem o uso da escala diagramática, foram significativamente diferentes de zero, exceto para o terceiro avaliador. Os demais avaliadores foram pouco acurados na avaliação da severidade da mancha alvo para as retas de regressão apresentando um valor médio deste parâmetro de 5,10 (Tabela 1). Estes avaliadores superestimaram esta doença e foram encontrados seis valores do coeficiente linear maior que zero. $\mathrm{O}$ oitavo subestimou a severidade real a níveis baixos de intensidade da mancha alvo com o intercepto igual $-1,90$.

A análise da acurácia, com o uso da escala, indicou valores da inclinação da reta não significativos para todos os avaliadores. Observando-se as estimativas dos coeficientes lineares ou intercepto $(a)$, seis avaliadores superestimaram a severidade da mancha alvo quando não utilizaram a escala, reduzindo para cinco avaliadores, quando utilizaram a escala. A análise de precisão, estimada pelo coeficiente de determinação $\left(R^{2}\right)$, indicou valores entre 0,63 e 0,89 , com média de 0,77 e desvio padrão 0,9 para a estimativa 


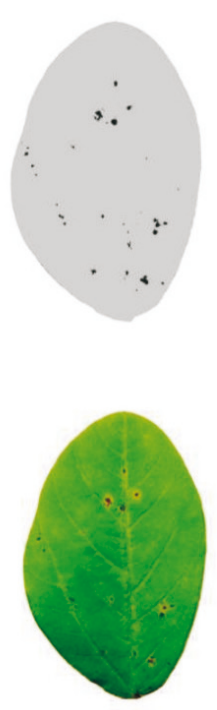

$1 \%$
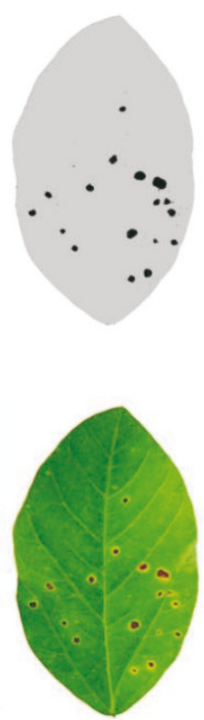

$2 \%$
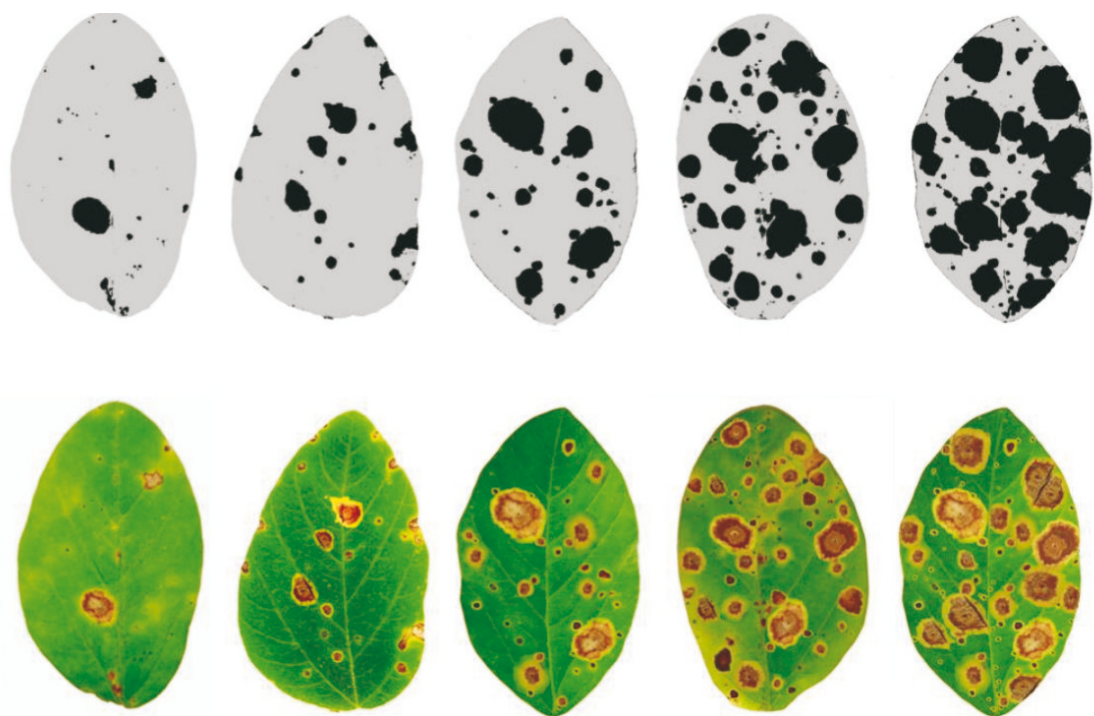

$5 \%$

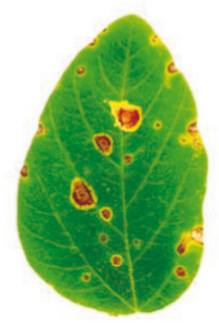

$9 \%$

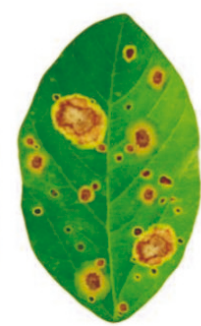

$19 \%$

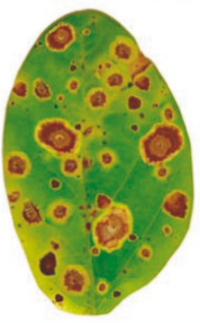

$33 \%$

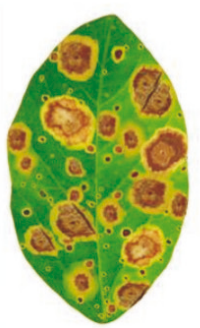

$52 \%$

FIGURA 1 - Escala diagramática para avaliação da severidade da mancha alvo da soja.

TABELA 1 - Valores estimados e teste $t$ para os parâmetros intercepto ou coeficiente linear $(a)$, coeficiente angular $(b)$, valores dos coeficientes de determinação $\left(\mathrm{R}^{2}\right)$ para o modelo de regressão linear comparando a severidade real da mancha alvo da soja, com a estimada, com o uso ou não da escala diagramática

\begin{tabular}{|c|c|c|c|c|c|c|c|c|c|c|}
\hline \multirow[t]{3}{*}{ Avaliador } & \multicolumn{5}{|c|}{ Sem escala } & \multicolumn{5}{|c|}{ Com escala } \\
\hline & \multicolumn{2}{|c|}{ Estimativa } & \multicolumn{2}{|c|}{$\begin{array}{c}\text { Valor do teste } t \text { para } 0 \\
\text { parâmetro }\end{array}$} & \multirow{2}{*}{$\begin{array}{c}\begin{array}{c}\text { Coef. de } \\
\text { Determ. }\end{array} \\
\mathbf{R}^{2}\end{array}$} & \multicolumn{2}{|c|}{ Estimativa } & \multicolumn{2}{|c|}{$\begin{array}{c}\text { Valor do teste } t \text { para } 0 \\
\text { parâmetro }\end{array}$} & \multirow{2}{*}{$\begin{array}{c}\begin{array}{c}\text { Coef. } d \\
\text { Determ }\end{array} \\
R^{2}\end{array}$} \\
\hline & $a$ & $b$ & $a$ & $b$ & & $a$ & $b$ & $a$ & $b$ & \\
\hline 1 & $2,55^{*}$ & 0,92 & $2,83^{*}$ & $-1,41$ & 0,89 & $-0,93$ & 1,03 & 1,85 & 1,17 & 0,97 \\
\hline 2 & $2,87^{*}$ & 1,01 & $2,36^{*}$ & 0,13 & 0,84 & $1,83^{*}$ & 1,03 & $2,23^{*}$ & 0,66 & 0,92 \\
\hline 3 & $-0,08$ & 0,99 & 0,06 & $-0,013$ & 0,84 & 0,80 & 1,03 & 0,85 & 0,47 & 0,90 \\
\hline 4 & $8,71^{*}$ & 1,03 & $4,77^{*}$ & $-0,96$ & 0,71 & $3,19^{*}$ & 1,12 & $2,21^{*}$ & 1,45 & 0,82 \\
\hline 5 & $11,17^{*}$ & $1,77^{*}$ & $3,88^{*}$ & $4,55^{*}$ & 0,74 & $2,76^{*}$ & 1,07 & $2,01^{*}$ & 0,86 & 0,82 \\
\hline 6 & $6,82^{*}$ & 1,06 & $3,17^{*}$ & 0,46 & 0,65 & $7,25^{*}$ & 0,97 & $4,60^{*}$ & $-0,35$ & 0,74 \\
\hline 7 & $10,70^{*}$ & 0,98 & $5,14^{*}$ & $-0,021$ & 0,63 & $5,62^{*}$ & 0,93 & $3,15^{*}$ & $-0,70$ & 0,67 \\
\hline 8 & $-1,90^{*}$ & $0,78^{*}$ & $2,19^{*}$ & $4,38^{*}$ & 0,86 & 1,12 & 0,89 & 1,06 & $-1,72$ & 0,84 \\
\hline Médias & 5,10 & 1,06 & & & 0,77 & 2,70 & 1,01 & & & 0,84 \\
\hline
\end{tabular}

*A hipótese de nulidade ( $a=0$ ou $b=1)$ foi rejeitada pelo teste $\mathrm{t}(p \leq 0.05)$, ou seja, valores para o teste t são significativos ao nível de significância escolhido. Os valores críticos para o teste t ao nível de $5 \%$ e $1 \%$ com 38 graus de liberdade são os que seguem: $t_{(0.05 ; 38 g l)}=2.024$ e $t_{(0.01 ; 38 g l)}=2.712$

sem escala e, valores entre 0,67 e 0,97 , com média de 0,84 e desvio padrão 0,9 para a estimativa com escala. As estimativas sem e com escala, explicaram $77 \%$ e $84 \%$ da variação na mensuração da doença, respectivamente. Detectou-se maior confiabilidade na avaliação da doença com a escala, pois houve aumento expressivo nos valores dos coeficientes $\mathrm{R}^{2}$, com exceção do avaliador oito, mas não prejudicando esta fidedignidade ( $\left.\hat{\mathrm{a}}=1,12^{\mathrm{ns}} \mathrm{e} \mathrm{b}=0,89^{\mathrm{ns}}\right)$.

Se verificarmos os resíduos obtidos pela análise de regressão, entre a severidade avaliada e a real, sem e com a escala diagramática, pode-se fazer as seguintes interpretações: o primeiro avaliador sem o uso da escala, superestimou em 23 resíduos (57,5\%) e subestimou em 17 resíduos (42,5\%). A dispersão destes erros ocorreu entre $-9,05$ e 9,50 (Tabela 2). Da mesma forma, utilizando a escala, houve super e subestimação em 22 e 18 resíduos, respectivamente, mas houve redução na magnitude destes desvios sendo o intervalo de variação para os mesmos entre $-6,77$ e 4,11 (Tabela 2 e Figura 2). Dentre todos os avaliadores, sem a utilização da escala diagramática, foi encontrada a menor estimativa da variância dos erros para $\mathrm{o}$ avaliador oito $\left(\hat{\sigma}_{\text {Erros }}^{2}=13,727\right)$. A maior variabilidade, nesta mesma situação ocorreu para o avaliador cinco $\left(\hat{\sigma}_{\text {Erros }}^{2}=151,882\right)$ e este último, usando a escala, teve redução em mais ou 


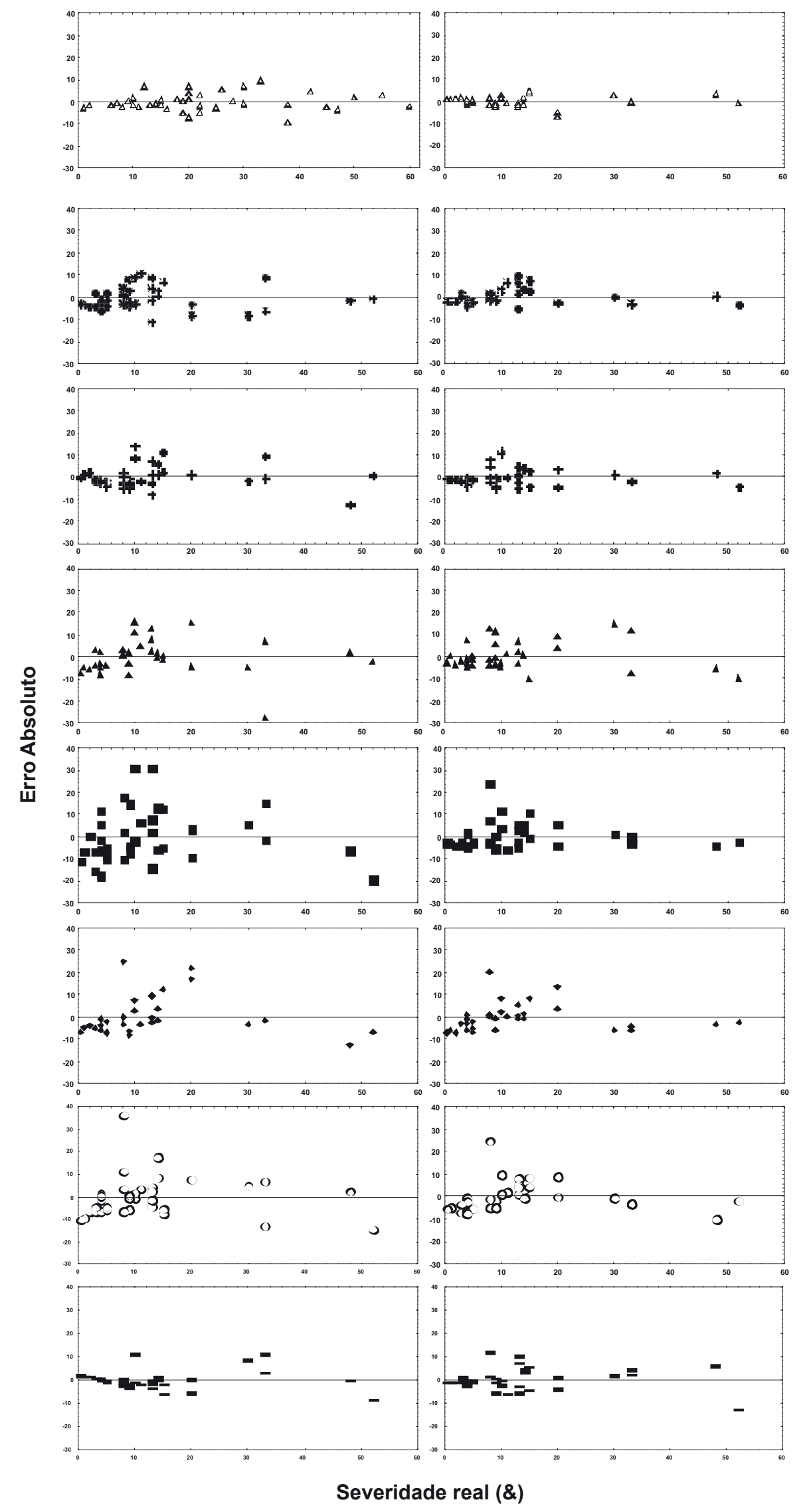

FIGURA 2 - Erros absolutos (severidade estimada menos a severidade real) das avaliações da mancha alvo de oito avaliadores, sem (esquerda) e com (direita) auxílio da escala diagramática. Avaliadores: $\Delta=1, *=2,+=$ $3, \boldsymbol{\Delta}=4, \boldsymbol{\square}=5, \bullet=6, \circ=7$ $\mathrm{e}-=8$. 
menos quatro vezes $\left(\hat{\sigma}_{\text {Erros }}^{2}=34,374\right)$. O intervalo dos resíduos, sem o uso da escala, variou de $-19,36$ a 31,09, apresentando uma amplitude de 50,45 reduzindo para 29,21 com o uso da escala (Tabela 2).

A constatação da tendência dos avaliadores superestimarem a severidade da doença, também foi verificada em estudos semelhantes com outros patossistemas (Sherwood et al., 1983; Parker et al., 1995; Godoy et al., 2006). No entanto, esta tendência talvez não seja tão acentuada para a mancha alvo, porque as lesões são relativamente grandes quando comparadas a outras doenças foliares da soja e, segundo Sherwood et al. (1983), a percepção humana costuma superestimar a severidade em uma área com maior número de lesões pequenas, do que em uma mesma área mas com menor número de lesões grandes. Conclui-se que a utilização da escala diagramática desenvolvida resultou em melhor acurácia e precisão das estimativas realizadas, sendo uma ferramenta de fácil e rápida utilização, e pode ser adotada para auxiliar na avaliação da mancha alvo da soja em diversos experimentos, com diferentes propósitos.

TABELA 2 - Intervalo de variação dos erros ( [ $]_{\text {Erros }}$ ), estimativas dos parâmetros obtidas por meio dos resíduos da análise de regressão linear $\left(\hat{\sigma}_{\text {Erros }}^{2}, \hat{\sigma}_{\text {Erros }}, \bar{X}_{\text {Erros }}\right)$, número de avaliações superestimadas e subestimadas, sem e com auxílio da escala diagramática para avaliar severidade da mancha alvo da soja

\begin{tabular}{|c|c|c|c|c|c|c|c|}
\hline \multicolumn{2}{|c|}{ AVALIADORES } & \multicolumn{4}{|c|}{ ERROS } & \multirow[t]{2}{*}{ SUPER } & \multirow[t]{2}{*}{ SUB } \\
\hline & & {[]$_{\text {Erros }}$} & $\hat{\sigma}_{\text {Erros }}^{2}$ & $\hat{\sigma}_{\text {Erros }}$ & $\bar{X}_{\text {Erros }}$ & & \\
\hline \multirow[t]{2}{*}{1} & Sem Escala & $-9,05 ; 9,50$ & 14,840 & 3,852 & 0 & 23 & 17 \\
\hline & Com Escala & $-6,77 ; 4,11$ & 4,602 & 2,145 & $5 * 10^{-5}$ & 22 & 18 \\
\hline \multirow[t]{2}{*}{2} & Sem Escala & $-10,99 ; 11,02$ & 27,111 & 5,206 & $1,75^{*} 10^{-4}$ & 24 & 16 \\
\hline & Com Escala & $-5,24 ; 9,75$ & 12,240 & 3,498 & $-25^{*} 10^{-6}$ & 25 & 15 \\
\hline \multirow[t]{2}{*}{3} & Sem Escala & $-12,25 ; 14,21$ & 25,043 & 5,004 & $15,1 * 10^{-3}$ & 23 & 17 \\
\hline & Com Escala & $-5,14 ; 11,93$ & 16,069 & 4,008 & $-25 * 10^{-6}$ & 27 & 13 \\
\hline \multirow[t]{2}{*}{4} & Sem Escala & $-27,71 ; 15,98$ & 61,126 & 7,818 & $-75^{*} 10^{-6}$ & 21 & 19 \\
\hline & Com Escala & $-1004 ; 1510$ & 37885 & 6155 & 0 & 24 & 16 \\
\hline \multirow[t]{2}{*}{5} & Sem Escala & $-19,36 ; 31,09$ & 151,882 & 12,324 & $150 * 10^{6}$ & 22 & 18 \\
\hline & Com Escala & $-5,53 ; 23,68$ & 34,374 & 5,863 & $175^{*} 10^{-6}$ & 25 & 15 \\
\hline \multirow[t]{2}{*}{6} & Sem Escala & $-12,67 ; 24,71$ & 84,531 & 9,194 & $175^{*} 10^{-6}$ & 30 & 10 \\
\hline & Com Escala & $-7,24 ; 20,01$ & 45,471 & 6,743 & $75 * 10^{-6}$ & 24 & 16 \\
\hline \multirow[t]{2}{*}{7} & Sem Escala & $-14,58 ; 36,47$ & 79,360 & 8,908 & $75^{*} 10^{-6}$ & 20 & 20 \\
\hline & Com Escala & $-10,05 ; 24,97$ & 58,168 & 7,626 & $-50 * 10^{-6}$ & 27 & 13 \\
\hline \multirow[t]{2}{*}{8} & Sem Escala & $-8,46 ; 11,29$ & 13,727 & 3,705 & $25 * 10^{-6}$ & 23 & 17 \\
\hline & Com Escala & $-12,54 ; 11,73$ & 20,563 & 4,534 & $-75^{*} 10^{-6}$ & 23 & 17 \\
\hline
\end{tabular}

\section{REFERÊNCIAS BIBLIOGRÁFICAS}

Almeida AMR, Machado CC, Ferreira LP, Lehman OS, Antonio H (1976) Ocorrência de Corynespora cassicola no Estado de São Paulo. Fitopatologia Brasileira 1:111-112.

Almeida AMR, Ferreira LP, Yorinori JT, Silva JFV, Henning AA (1997) Doenças da Soja (Glycine max L.). In: Kimati H, Amorim L, Bergamin Filho A, Camargo LEA, Rezende L (Eds.) Manual de Fitopatologia. Vol. 2. Doenças das Plantas Cultivadas. São Paulo SP. Ceres. pp. 376-399.

Angelotti F, Scapin CR, Tessmann DJ, Vida JB, Oliveira RR, Canteri MG (2008) Diagrammatic scale for assessment of grapevine rust. Tropical Plant Pathology 33:439-443.

Bergamin Filho A, Amorim L (1996) Doenças de plantas tropicais: epidemiologia e controle econômico. São Paulo SP. Agronômica Ceres.
Charnet R, Freire CAL, Charnet EMR, Bonvino H (1999) Análise de Modelos de Regressão Linear com Aplicações. Campinas SP. Editora Unicamp.

Godoy CV, Koga LJ, Canteri MG (2006) Diagrammatic scale for assessment of soybean rust severity. Fitopatologia Brasileira 31:63-68.

Henning AA, Almeida AMR, Godoy CV, Seixas CDS, Yorinori, JT, Costamilan LM, Ferreira LP, Meyer MC, Soares RM, Dias WP (2005) Manual de identificação de doenças de soja. Londrina: Embrapa Soja (Embrapa Soja Documentos 256).

Nutter Jr FW, Schultz PM (1995) Improving the accuracy and precision of disease assessments: selection of methods and use of computer-aided training programs. Canadian Journal of Plant Pathology 17:174-184.

Parker SR, Shaw MW, Royle DJ (1995) The reliability of visual estimates of disease severity on cereal leaves. Plant Pathology 44:856-864. 
Sherwood RT, Berg CC, Hoover MR, Zeiders KE (1983) Illusions in visual assessment of stagonospora leaf spot of orchardgrass. Phytopathology 73:173-177.

Tecnologias de Produção de Soja, Região Central do Brasil 2008 (2008) Londrina PR. Embrapa Soja, Embrapa Cerrados, Embrapa Agropecuária Oeste. Sistemas de Produção. Embrapa Soja no 12.
Vale FXR, Fernandes Filho EIF, Liberato JR (2003) QUANT. A software for plant disease severity assessment. $8^{\text {th }}$ International Congress of Plant Pathology, Christchurch New Zealand. p. 105.

Zonta EP, Machado AA, Silveira Júnior P (1982). Sistema de Análise Estatística- SANEST, Registro na SEI No. 066060. Pelotas RS. Universidade Federal de Pelotas.

TPP 9025 - Recebido 13 Fevereiro 2009 - Aceito 16 Setembro 2009 Editor de Seção: Lilian Amorim 